\title{
A atuação do residente em Odontologia Hospitalar neonatal na abordagem multidisciplinar do SUS: relato de experiência
}

Nayara Silva de Gouvêa*; Jessyca Twany Demogalski*; Marcos Cezar Pomini**; Caique Mariano

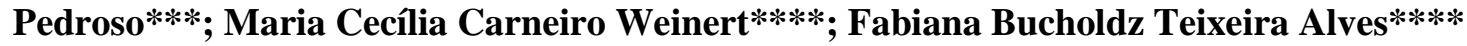

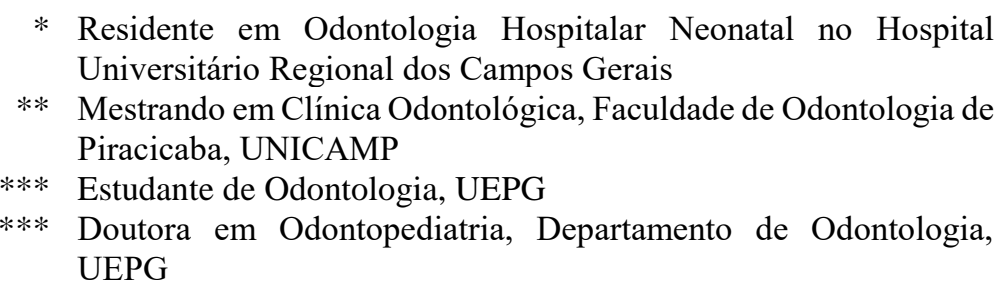

Recebido em 11/01/2018. Aprovado em 16/11/2018.

\begin{abstract}
RESUMO
A reorganização dos serviços de saúde vinculada ao programa Humaniza SUS possibilita o cuidado multiprofissional do indivíduo. Neste sentido, a inclusão do cirurgião-dentista nas equipes visa somar esforços para o alcance da integralidade da atenção, a qual abrange as diversas alterações que podem acometer o sistema estomatognático de sujeitos em cuidados hospitalares. A residência em odontologia hospitalar neonatal, como parte integradora da abordagem multiprofissional, é extremamente importante para garantir a abordagem adequada de gestantes, puérperas e recémnascidos $(\mathrm{RN})$ em sua plenitude. As frentes de atuação do residente em odontologia neonatal envolvem, principalmente, o pré-natal odontológico, o puerpério imediato e mediato, a abordagem ambulatorial e as unidades de terapia intensiva neonatal e pediátrica. Este artigo objetiva relatar a experiência de atuação de residentes em odontologia hospitalar neonatal em um hospital escola pertencente ao Sistema Único de Saúde (SUS) do Paraná.

Descritores: Saúde Bucal. Neonatologia. Internato e Residência. Sistema Único de Saúde.
\end{abstract}

\section{INTRODUÇÃO}

Atualmente, os serviços de saúde e as políticas governamentais têm exigido dos profissionais uma abordagem centrada na humanização e na integralidade do ser humano. No que tange ao ensino odontológico, as Diretrizes Curriculares Nacionais (DCN) enfatizam a necessidade da formação em consonância com essas demandas ${ }^{1}$. A Odontologia Hospitalar frequentemente assume o papel de abordagem 
global do sujeito, uma vez que suas ações, preventivas ou curativas, estão voltadas aos cuidados das alterações bucais que se relacionam com a saúde geral do indivíduo, exigindo procedimentos de baixa, média ou alta complexidade $^{1,2}$.

Tendo em vista que nenhuma categoria profissional isoladamente possui o conhecimento requerido para atender todas as necessidades de um indivíduo, a atuação em equipes multiprofissionais é uma realidade que assegura a abordagem holística que deve ser oferecida ao paciente hospitalizado $^{3}$. Nesse sentido, a inserção do cirurgião-dentista (CD) na equipe hospitalar tem contribuído para a melhora da qualidade de vida do indivíduo e para a redução no tempo de internação e utilização de medicamentos, logo, no custo geral do tratamento ${ }^{3,4}$. As atividades odontológicas em nível hospitalar envolvem particularmente o estabelecimento de protocolos para a evolução do cuidado em higiene oral, o diagnóstico de alterações bucais, a resolução de urgências e emergências e o controle e a prevenção de bacteremias e alterações da microbiota. $\mathrm{O}$ campo de atuação envolve, usualmente, ambulatórios, leitos, centros cirúrgicos e unidades de terapia intensiva $(\mathrm{UTI})^{3,4}$.

A reorganização dos serviços de saúde frente ao programa Humaniza SUS possibilita uma intervenção multiprofissional para a escuta e resolução das dificuldades encontradas na abordagem em saúde, tendo a humanização como eixo norteador, consolidando a gestão participativa e facilitando o acesso dos usuários e a educação permanente dos profissionais ${ }^{5}$. Com essa reestruturação, espera-se que a odontologia neonatal hospitalar, inserida em um cenário de residência multiprofissional, concretize a abordagem biopsicossocial e integral maternoinfantil, possibilitando melhora da qualidade de vida do binômio mãe-filho.

A atuação do $\mathrm{CD}$ em equipes multipro- fissionais de atenção hospitalar materno-infantil é uma experiência recente, com poucos relatos na literatura elucidando suas estratégias. Contudo, algumas instituições já a executam há um maior tempo. Uma dessas experiências é encontrada no Hospital São Francisco de Assis, da cidade de Tupã/SP, onde o serviço de odontologia neonatal hospitalar é desenvolvido desde $1997^{6}$. Vale salientar que as ações em saúde dirigidas à gestante e ao recém-nascido $(\mathrm{RN})$ constituem-se como importante estratégia preventiva de saúde bucal a curto e longo prazos para o núcleo familiar? ${ }^{7}$.

A neonatologia, como uma especialidade dedicada à assistência ao RN, à gestante e à puérpera, possui sua principal meta centrada na redução da morbimortalidade perinatal e na sobrevivência do RN nas melhores condições funcionais possíveis ${ }^{8}$. A preocupação com a saúde perinatal tem sido preconizada nas estratégias do Ministério da Saúde no Brasil ${ }^{4}$, uma vez que o componente neonatal é um dos grandes desafios na redução da mortalidade infantil. Nesse sentido, o Brasil tem firmado compromissos e estratégias internas e externas para a melhoria da qualidade da atenção à saúde no período gravídico-puerperal ${ }^{9}$. As estratégias destinadas à melhoria da qualidade da assistência neonatal devem incluir todas aquelas voltadas às políticas assistenciais, no sentido de humanizar o processo de nascimento, acentuando a qualidade da assistência técnica oferecida a essa população (mulher, recém-nascido e família) e a abordagem fundamentada na integralidade do ser.

A proposta da residência multiprofissional em Neonatologia visa, além da formação de um profissional qualificado às exigências do Sistema Único de Saúde (SUS), a formação de um cidadão com pensamento crítico que busque, em seus espaços de atuação profissional, social e política, a possibilidade de construir coletivamente soluções aos problemas que acometem os usuários. Assim, este trabalho tem por objetivo relatar a experiência da atuação de residentes em Odontologia 
Hospitalar neonatal em um hospital escola no Paraná.

\section{RELATO DE EXPERIÊNCIA}

\section{Campos de atuação do residente em Odontologia} Hospitalar neonatal

Dentro de um complexo hospitalar, o residente em Odontologia Hospitalar neonatal pode atuar em diversos cenários de prática, como:

1. Pré-natal - educação das gestantes estratificadas em alto risco sobre a importância do pré-natal odontológico na prevenção de alterações bucais e sistêmicas, benefícios do aleitamento materno exclusivo, mudanças de hábitos alimentares, orientações de higiene bucal, bem como o atendimento ambulatorial odontológico propriamente dito.

2. Puerpério imediato - acompanhamento materno a fim de auxiliar o contato pele a pele (criação de vínculo afetivo entre a mãe e o bebê) e estimular a sucção imediata, enfatizando a importância do aleitamento materno exclusivo.

3. Puerpério mediato - triagem para o diagnóstico de alterações bucais, realização do teste da linguinha (TL), cirurgia de frênulo lingual (frenotomia) e condução no manejo da pega correta para a amamentação, visando prevenção de fissuras mamárias e desmame precoce.

4. Ambulatório odontológico - avaliações odontológicas agendadas e acompanhamento do recém-nascido até a faixa etária de dois anos de idade, visando sobretudo promoção de saúde bucal.

5. UTI neonatal (UTIneo) e pediátrica (UTIped) protocolos de higiene bucal, via Bundle, para redução dos índices de pneumonia associada à ventilação mecânica (PAV), diagnóstico de anormalidades do sistema estomatognático e auxílio no aleitamento materno.

Em todos os campos de prática, o residente possibilita a confecção e padronização de
Procedimentos Operacionais Padrão (POPs) e materiais de orientação, assim como a capacitação e supervisão da equipe multiprofissional no que tange a higiene bucal dos RN.

\section{Atuação no pré-natal}

A promoção de saúde bucal é parte fundamental da saúde integral materno-infantil. $\mathrm{O}$ exercício do residente em Odontologia Hospitalar neonatal, no cenário de prática clínica durante o pré-natal busca, sobretudo, a conscientização materna sobre a importância do acompanhamento odontológico no período gravídico-puerperal, visando à promoção de saúde e estabelecimento de vínculo entre $\mathrm{CD}$ e gestantes. Neste cenário, destaca-se a importância dos cuidados com a sua saúde bucal e sua relação com a saúde e o desenvolvimento do bebê, salientando que, mesmo que as evidências científicas não indiquem claramente a relação entre doença periodontal e parto prematuro/baixo peso ao nascimento ${ }^{10,11}$, a educação e prevenção de problemas bucais podem garantir uma redução no número de microrganismos bucais, consequentes mediadores de inflamação. É importante salientar que o prénatal odontológico é um momento-chave para manter ou resgatar a saúde bucal por meio de medidas preventivas e/ou curativas, minimizando possíveis alterações indesejáveis no nascimento e desenvolvimento do bebê $\hat{e}^{12}$.

A atuação dos residentes no pré-natal conta com a participação de estudantes de graduação do curso de Odontologia, por meio de ações extensionistas $^{13}$. Em conjunto, residentes e acadêmicos desenvolveram um protocolo de abordagem, uma ficha de anamnese e materiais educativos para as orientações. Os assuntos abordados nesta etapa essencialmente abrangem a desmistificação de crenças e preocupações sobre a gravidez e tratamento odontológico; relação do estilo de vida e hábitos com a saúde e o desenvolvimento do bebê; conscientização acerca 
de problemas bucais; informação sobre os efeitos de uso de drogas, cigarro e álcool; importância da higiene bucal no controle de biofilme, especialmente durante a gestação devido as alterações hormonais que podem favorecer $o$ desenvolvimento de doenças periodontais; e importância de hábitos alimentares saudáveis e aleitamento materno exclusivo ${ }^{13}$. Assim, a atuação do residente passa a contribuir para a difusão popular sobre a necessidade da consulta odontológica no pré-natal, subsidiada nas Políticas Nacionais de Saúde Bucal ${ }^{14}$.

\section{Atuação no puerpério imediato}

A humanização da assistência ao parto implica principalmente que a atuação do profissional respeite os aspectos de sua fisiologia, não intervenha desnecessariamente, reconheça os aspectos sociais e culturais do parto, assim como ofereça o necessário suporte emocional à mulher e sua família, promovendo o vínculo mãe-bebê ${ }^{15,16}$. Da mesma maneira, a presença do $\mathrm{CD}$ no pré e pós-parto imediato é essencial para a formação do vínculo profissional-indivíduo, visto que logo após o nascimento, o profissional pode auxiliar na identificação dos reflexos orais e, consequentemente no aleitamento materno.

A amamentação imediatamente após o parto é recomendada pela Organização Mundial da Saúde (OMS). Essa estratégia é considerada prioritária para a promoção, proteção e apoio ao aleitamento materno e baseia-se na capacidade da interação RN-mãe nos primeiros minutos de vida. Esse contato inicial é fundamental para o estabelecimento do vínculo binomial, além de aumentar a duração do aleitamento materno ${ }^{17}$. Desta maneira, os $\mathrm{RN}$ são colocados imediatamente em contato com o seio materno para promoção da amamentação. Assim, o contato pele a pele é incentivado (para mães e RNs estáveis), o qual resulta em numerosos benefícios para a saúde materno-infantil, como estabilização dos níveis de glicose e regulação da temperatura corporal do bebê.

\section{Atuação no puerpério mediato}

No puerpério mediato, ou seja, no leito, é realizado o exame físico extra e intrabucal do RN, visando diagnosticar principalmente alterações bucais e de inserção do frênulo lingual, os quais podem influir sobre a efetivação da amamentação. O teste da linguinha (TL) tornou-se obrigatório em todos os hospitais e maternidades públicas e privadas e visa à identificação precoce dos casos de anquiloglossia $^{18}$. Essa alteração é uma anomalia congênita e caracteriza-se pela presença do frênulo lingual anormalmente curto, o qual pode restringir, em diferentes graus, os movimentos linguais. As variações na inserção do freio lingual podem acarretar distúrbios da fala, dentição, deglutição e dificuldade de sucção, além de problemas psicológicos entre a mãe e o bebê durante o aleitamento materno ${ }^{19,20}$.

O TL é realizado antes da alta hospitalar do $\mathrm{RN}$ por meio de um fluxograma estabelecido pela equipe, o qual é baseado no protocolo de Bristol Tongue Assessement Tool (BTAT), conforme a Nota Técnica ${ }^{\circ} 09 / 20161^{21}$. Nos casos mais graves, a abordagem cirúrgica de liberação do freio lingual (frenotomia) é realizada antes da alta hospitalar e o $\mathrm{RN}$ é agendado para acompanhamento no ambulatório de Saúde Bucal Materno-Infantil (SBMI) do hospital. A frenotomia é uma proposta cirúrgica segura, prática e eficaz no tratamento da anquiloglossia em bebês. $\mathrm{O}$ procedimento consiste em anestesia tópica local com uma incisão horizontal do freio lingual, a fim de permitir a mobilidade lingual ${ }^{19,20}$. Nos casos moderados e leves a reavaliação é agendada para o $10^{\circ}$ dia de vida do RN.

No leito, o residente orienta e auxilia no manejo da pega correta e orientações sobre o aleitamento materno, especialmente nos casos de imaturidade anatomofisiológica. Os residentes, 
neste cenário, desenvolveram materiais ilustrativos para instrução das mães sobre a importância do aleitamento materno exclusivo no desenvolvimento do sistema estomatognático do bebê e os malefícios da sucção não-nutritiva.

\section{Atuação no ambulatório odontológico}

O ambulatório de SBMI, como parte integrante da Residência Multiprofissional em Neonatologia, centra suas ações na atenção primária em saúde oral e busca ampliar o atendimento dos bebês nascidos no hospital, sendo o acompanhamento realizado até os 24 meses de idade. O principal objetivo do acompanhamento é a promoção de saúde bucal na primeira infância, ressaltando os aspectos educativo-preventivos. A medida que os bebês são atendidos, os conhecimentos são reforçados aos pais e responsáveis, uma vez que a conscientização do núcleo familiar na primeira infância é fundamental para a eliminação de hábitos inadequados e a prevenção de problemas futuros. O retorno tem se mostrado satisfatório, pois nasce de um desejo natural dos pais de oferecerem o melhor para seus filhos. Vale ressaltar que a educação dos sujeitos é um dos principais componentes da filosofia preventiva. Pode-se afirmar que a prevenção é primordialmente educação, a qual tem potencial de guiar os indivíduos à adoção de práticas indispensáveis e assumirem responsabilidades frente à manutenção da saúde bucal do núcleo familiar $^{22}$.

No ambulatório, os atendimentos se estendem com sessões no $10^{\circ}$ dia após o nascimento, nas quais são avaliados os $\mathrm{RN}$ agendados para retorno (presença de anquiloglossia de grau leve/moderado ou dificuldade no aleitamento materno até a alta hospitalar). Neste momento, os RN são reexaminados por meio do BTAT. Nesta ocasião, além do exame clínico bucal, a importância do aleitamento materno exclusivo, auxílio na pega- correta e esclarecimento de dúvidas das puérperas são reforçados. Os RN que receberam frenotomia são agendados para acompanhamento após sete dias. Em todos os retornos ao hospital, os residentes realizam o exame clínico da cavidade bucal procurando identificar anormalidades em dentes, gengivas e nas funções relacionadas à face, como sucção, respiração e deglutição.

Aos três meses de idade do lactente a mãe participa de uma palestra do projeto Saúde Bucal Materno-Infantil, na qual recebe reforço sobre o aleitamento materno e higiene oral do bebê, irrupção dentária, hábitos de sucção não-nutritivos, introdução desnecessária do açúcar, hábitos nutricionais saudáveis e doença cárie. Aos seis meses o atendimento torna-se clínico, visando à prevenção e ao acompanhamento odontológico. Os retornos são realizados aos 6, 12, 18 e 24 meses de idade e têm como eixo norteador que a intervenção preventiva traz inquestionáveis benefícios para a qualidade de vida dos indivíduos.

Atuação na unidade de terapia intensiva neonatal (UTIneo) e pediátrica (UTIped)

A prática hospitalar do CD em UTIneo/ped contempla as necessidades de atendimento do indivíduo, cujo estado exige assistência e observação contínua dos profissionais de saúde devido à instabilidade do quadro clínico ${ }^{23}$. Durante a permanência na UTIneo/ped, é comum a ocorrência de alterações no sistema estomatognático associadas às manifestações de doenças sistêmicas ou ao estado geral de saúde do sujeito, ao uso de medicamentos, ou ainda à presença de equipamentos como o tubo do respirador artificial. Assim, estes fatores justificam a importância do $\mathrm{CD}$ na equipe multiprofissional neonatal em benefício do paciente em estado crítico $^{11}$.

Os RN em UTIneo apresentam risco elevado de colonização por microrganismos patogênicos. Dentre os fatores de risco, relacionados com a 
bacteremia nosocomial em $\mathrm{RN}$, encontram-se a imaturidade do sistema imunológico, o baixo peso, a necessidade frequente de procedimentos invasivos e ventilação mecânica, assim como fatores associados ao ambiente e aos profissionais da saúde $^{24}$. Uma das principais infecções em unidades de terapia intensiva associada a assistência à saúde do indivíduo (IRAS) é a PAV ${ }^{25}$. Esta alteração consiste em uma infecção gerada por microrganismos que são disseminados para o parênquima pulmonar após 48 horas ou mais de intubação orotraqueal em UTI, causando alteração local e sistêmica ${ }^{26,27}$.

Com o intuito de reduzir os índices de PAV, instituiu-se manejo de cuidados de higiene oral para neonatos sob ventilação mecânica, focado na prática do cuidado, uma vez que cuidados bucais protocolados tendem a diminuir os quadros de infecções por microrganismos e melhorar a sobrevida dos indivíduos ${ }^{24}$. Para tal, seguindo o proposto por Weber $(2016)^{26}$, realiza-se a limpeza da cavidade oral com gaze estéril embebida em água destilada, seguida pela aspiração orotraqueal. Essa técnica tem se mostrado efetiva na redução da disseminação de microrganismos para as vias aéreas inferiores. Nesse sentido, é importante ressaltar que a ventilação mecânica afeta o fluxo salivar, usualmente ressecando mucosas, contribuindo assim para mucosites, colonização de bactérias gram-negativas e surgimentos de fissuras labiais, portas de entrada para microrganismos ${ }^{24}$. Tem-se por protocolo a hidratação das mucosas em processo de ressecamento, por meio do uso de óleos a base de ácidos graxos. Já para as crianças em UTIped sob ventilação espontânea e alimentação por via oral, faz-se uso de gaze estéril ou swab embebido em solução não alcoólica de gluconato de clorexidina $0,12 \%$. A fricção é realizada suavemente no fundo de vestíbulo, mucosas jugais e língua, podendo ser complementada por escova dental para remoção de restos alimentares aderidos nas superfícies dentárias.

A atuação do residente em Odontologia Hospitalar neonatal na UTIneo e UTIped é multiprofissional, com ênfase no relacionamento interprofissional, realizando diagnósticos de alterações da cavidade oral, além do estabelecimento de protocolos de higiene oral. Neste sentido, é importante salientar que a higiene oral por si só não gera redução nos índices de PAV em UTI, necessitando de um apanhado de atividades (bundle), desenvolvidas em conjunto pela equipe multiprofissional, para resultar em mudanças significativas ${ }^{26,27}$. A incorporação do bundle, composto por higiene das mãos, higiene oral, aspiração orotraqueal, posicionamento e hidratação de mucosas, visa à redução na disseminação de bactérias para vias aéreas inferiores causando possíveis bacteremias ${ }^{28}$.

\section{A experiência}

A atuação da equipe hospitalar multiprofissional do Hospital Universitário Regional dos Campos Gerais (HURCG), composta por profissionais de Enfermagem, Odontologia, Fisioterapia, Farmácia, Análises Clínicas, Assistência Social, Medicina, Nutrição, Fonoaudiologia e Psicologia, oferece uma vivência diferenciada da prática odontológica rotineira. A formação do CD é majoritariamente centrada na formação biologicista, tecnicista, curativa e individualista, voltada ao atendimento ambulatorial $^{29}$, o que gera uma das barreiras para a sua atuação na atenção em saúde no ambiente hospitalar em equipe multiprofissional.

As atividades da residência em Odontologia neonatal englobam a atuação em ambulatório de gestantes de alto risco no pré-natal, na UTIneo/ ped, centro cirúrgico e/ou sala de parto, alojamento conjunto (puerpério e unidade Canguru), unidade de cuidados intermediários neonatais e ambulatório clínico pós-natal. A atuação nesses setores exige do CD competências básicas e complexas, que muitas 
vezes não são abordadas em seu processo de formação, incluindo o manejo de gestantes, RN e puérperas. Assim, a residência exige do $\mathrm{CD}$ constante aprofundamento científico e clínico, que por sua vez oferece a atenção odontológica a um público que frequentemente é apontado na literatura como resistente à tal prática ${ }^{30}$.

Da mesma maneira, a formação do $\mathrm{CD}$ em geral não privilegia o manejo da interdisciplinaridade na atenção ao indivíduo. É importante salientar que o cuidado multiprofissional não é sinônimo de interdisciplinar. No primeiro, ocorre a atuação conjunta de disciplinas de forma isolada, enquanto a segunda oferece a troca de conhecimento e experiências entre os profissionais $^{31}$. Portanto, a atuação tem sido uma experiência enriquecedora, pois permite a desconstrução da ideia do atendimento prioritariamente isolado dos profissionais de uma equipe multiprofissional, acentuando a interlocução entre as áreas e as relações interprofissionais, as quais propiciam a construção do saber coletivo e agregação do conhecimento de cada categoria em um caso.

Neste sentido, os residentes apontam que o aprendizado para o manejo interprofissional é fruto da residência multiprofissional, uma vez a visão equivocada do atendimento isolado de cada categoria é suprimido pela experiência. As relações interprofissionais na residência neonatal do HURCG são cotidianas e podem ser vistas no esforço de correlacionar as disciplinas e os profissionais envolvidos, não somente por uma síntese isolada de uma situação clínica por diferentes especialidades, mas por uma abordagem única, centrada em uma proposição integrada, em que a resolução da situação clínica é o objetivo comum de toda a equipe. Um dos exemplos pode ser encontrado no ambulatório de gestantes de alto risco, onde a equipe multiprofissional atua de maneira a garantir a adesão das gestantes ao prénatal odontológico, uma vez que a discussão sobre a importância dos cuidados de saúde bucal durante a gestação é frequente. Assim, o entendimento da equipe multiprofissional é fundamental para que ocorra a prática do ensino voltado à interdisciplinaridade.

Ademais, nota-se que existe uma resistência dos profissionais que possuem formação voltada ao ambiente hospitalar no que tange a inserção do CD neste cenário. Nesse sentido, a residência tem permitido, além do cuidado especializado do sistema estomatognático do $\mathrm{RN}$, a educação gradativa da equipe multiprofissional, por meio da demonstração prática/teórica da importância do exercício odontológico hospitalar, tendo com eixo norteador que o bom relacionamento da equipe, subsidiada no cuidado integral, é primordial para a melhora do quadro clínico do indivíduo ${ }^{32}$.

\section{CONSIDERAÇÕES FINAIS}

A atenção voltada à saúde bucal possui papel importantíssimo para os indivíduos que se encontram hospitalizados, portanto o $\mathrm{CD}$ precisa compreender os aspectos da Odontologia, fundamentando-os na atenção integral e humanizada. $\mathrm{O}$ residente em Odontologia neonatal possui atuação dentro de sua especificidade, unido a equipe multiprofissional, visando promover melhoria da qualidade do atendimento prestado ao binômio mãe-filho, consequentemente com reflexos positivos na qualidade de vida infantil e familiar. A residência em Odontologia Hospitalar neonatal oferece ao $\mathrm{CD}$ uma vivência diferenciada da realidade clínica ambulatorial, acentuando as relações interprofissionais, pois permite a interlocução e o reforço das informações centrados em uma proposição integrada das diferentes categorias profissionais, oferecendo atendimento integral e humanizado para gestantes, puérperas e RN nos três níveis de atenção à saúde.

\section{ABSTRACT \\ The multidisciplinary work of the resident in}


neonatal hospital dentistry in the Brazilian Healthcare System: an experience report

The restructuring of the national healthcare system in line with the HumanizaSUS program has enabled a multidisciplinary care of the individual. Consistent with this, the incorporation of dental professionals into healthcare teams aims to aggregate efforts to provide a comprehensive care, which includes the conditions affecting the stomatognathic system of hospitalized patients. The residency in neonatal hospital dentistry, as an integral part of the multidisciplinary approach, is extremely important to ensure a comprehensive care of pregnant and puerperal women and newborns. The activities of neonatal dental residents are focused on dental prenatal care, early and late puerperium, outpatient clinical care, and neonatal and pediatric intensive care units. This study aims to report the experience of residents in neonatal hospital dentistry in a school hospital of the Brazilian Healthcare System (SUS) in the state of Paraná.

Descriptors: Oral Health. Neonatology. Internship and Residency. Unified Health System.

\section{REFERÊNCIAS}

1. Porto AN, Segundo AS, Borges AH, Semenoff TADV, Miranda FP. Hospital Dentistry: a proposal of a new discipline. RSBO. 2012;9(1):119-22.

2. Ministério da Saúde. Secretaria de Atenção à Saúde. Política Nacional de Humanização - Formação e intervenção. 1 ed. Brasília (DF): MS; 2010.

3. Euzébio LF, Viana KA, Cortines AAO, Costa LR. Atuação do residente cirurgiãodentista em equipe multiprofissional de atenção hospitalar à saúde materno-infantil. Rev Odontol Brasil Central 2013;22(60):1620.

4. Costa LRRS, Zardetto C, Araujo AMPG, Pavez CE, Torres GR, Verdugo IA, et al. Presença do odontopediatra em ambiente hospitalar. Rev Odontopediatr Latinoam. 2014;4(2).

5. Lima ENA, Souza ECF. Percepção sobre ética e humanização na formação odontológica. RGO. 2010;58(2):231-8.

6. Carvalho GD, Chiaradia DL, Chiaradia R. Saúde oral e enfoque odontológico. In. Carvalho MR, Gomes CF. Amamentação: bases científicas. 4 ed. Rio de Janeiro: Guanabara Koogan; 2017.

7. Moimaz SAS, Rocha NB, Saliba O, Garbin CAS. O acesso de gestantes ao tratamento odontológico. Rev Odontol UNICID. 2007;19(1):39-45.

8. Carvalho M, Gomes MASM. A mortalidade do prematuro extremo em nosso meio: realidade e desafios. J Pediatr (Rio J). 2005;81(1Supl):S111.

9. Ministério da Saúde. Secretaria de Atenção à Saúde. Departamento de Ações Programáticas estratégicas. Atenção humanizada ao recém-nascido de baixo peso - Método Canguru: manual técnico. 2 ed., Brasília (DF): MS; 2013.

10. Polyzos NP, Polyzos IP, Zavos A, Valachis A, Mauri D, Papanikolaou EG, et al. Obstetric outcomes after treatment of periodontal disease during pregnancy: systematic review and meta-analysis. BMJ. 2010;341:c7017.

11. Santos PSS, Soares Junior LAV. Medicina Bucal - A prática na odontologia hospitalar. 1.ed. São Paulo: Santos; 2012, 315p.

12. Welgatch MKM, Samalea DMV. Atenção Odontológica às gestantes na Estratégia da Saúde da Família. Rev Divulg Téc-Científ do ICPG 2008;3(12):73-9.

13. Pomini MC, Gawlik AT, Pereira N, Santos AR, Santos BR, Demogalski JT, et al. Educação em saúde bucal a gestantes, puérperas e primeira infância: relato de atividade de extensão. Rev Bras Ext 
Universit. 2017;8(3):143-8.

14. Ministério da Saúde. Secretaria de Atenção à Saúde. Departamento de Atenção Básica. Diretrizes da Política nacional de saúde Bucal. 2004. [Acesso em 01 jul. 2017]. Disponível em: http://bvsms.saude. gov.br/bvs/publicacoes/politica_nacional_b rasil_sorridente.pdf.

15. Falcone VM, Mäder CVN, Nascimento CFL, Santos JMM, Nóbrega FJ. Atuação multiprofissional e a saúde mental de gestantes. Rev Saúde Pública. 2005;39(4):612-8.

16. Dias MAB, Domingues RMSM. Desafios na implantação de uma política de humanização da assistência hospitalar ao parto. Ciênc Saúde Coletiva. 2005;10(3):669-705.

17. Boccolini CS, Carvalho ML, Oliveira MIC, Vasconcellos AGG. Fatores associados à amamentação na primeira hora de vida. Rev Saúde Pública. 2010;45(1):69-78.

18. Ministério da Saúde. Lei $n^{\circ}$ 13.002. 2014 [internet]. Obriga a realização do protocolo de avaliação do frênulo da língua em bebês. 2014 [Acesso em 01 jul. 2017]. Disponível em: http://www.planalto.gov.br/ccivil_03/ Ato2011-2014/2014/Lei/L13002.htm

19. Silva PI, Vilela JER, Rank RCLC, Rank MS. Frenectomia lingual em bebê - relato de caso. Rev Bahiana Odonto. 2016;7(3):220-7.

20. Opara IP, Gabriel-Job N, Opara K. Neonates presenting with severe complications of frenotomy: a case series. J Med Case Rep. 2012;6(77):1-4.

21. Ministério da Saúde. Nota Técnica $N^{o}$ 09/2016. 2016. [Acesso em 30 jun. 2017]. Disponível em: http://www.redeblh.fiocruz. br/media/notatecn9_16.pdf.

22. Kuhn E, Wambier DS. Incidência de lesões de cárie em bebês após 15 meses de um programa educativo-preventivo. Pesq Bras Odontoped. 2007;7(1):75-81.
23. Pinheiro TS, Almeida TF. A saúde bucal em pacientes de UTI. Rev Bahiana Odonto. 2014;5(2):94-103.

24. Padovani MCRL, Souza SAB, Sant'Anna GR, Guaré RO. Protocolos de cuidados bucais na Unidade de Tratamento Intensivo (UTI) Neonatal. Rev Bras Pesq Saúde. 2012;14(1):71- 80.

25. Dal-Bó K, Silva RM, Sakae TM. Infecção hospitalar em uma unidade de terapia intensiva neonatal do Sul do Brasil. Rev Bras Ter Intensiva. 2012;24(4):381-5.

26. Weber CD, Applying adult ventilatorassociated pneumonia bundle evidence to the ventilated neonate CCNS-Neonatal, RNC. Adv Neonatal Care. 2016;16(3):17890.

27. Perugini MRE, Perugini VH, Figueira FD, Fontana LMS, Diniz JJ, Santos DL et al. Impacto de um bundle nas taxas de pneumonia associada à ventilação mecânica (PAV) em uma unidade de terapia intensiva pediátrica em Londrina-PR. Semina: Ciênc Biol Saúde. 2015;36(1) supl. 259-66.

28. Silva GS, Nascimento ERP, Salles RK. Bundle de prevenção da pneumonia associada à ventilação mecânica: uma construção coletiva. Texto Contexto Enferm, 2012;21(4): 837-44.

29. Grande IMP, Prochnow R, Saab R, Pizzatto E. Desafios na formação do cirurgiãodentista para o SUS. Rev ABENO. 2016;16(3):2-6.

30. Martins RFM, Azevedo JAP, Dourado CRL, Ribeiro CCC, Alves CMC, Thomaz EBAF. Oral health behaviors and dental treatment during pregnancy: a cross-sectional study nested in a cohort in Northeast Brazil. Pesq Bras Odontoped Clin Integr. 2014;14(1):511.

31. Mendes LC, Matos LP, Schindler MF, Tomaz M, Vasconcellos SC. Relato de 
experiência do primeiro ano da residência multiprofissional hospitalar em saúde, pela ótica da Psicologia. Rev SBPH. 2011;14(1):125-41.
32. Amaral COF, Marques JA, Bovolato MC, Parizi AGS, Oliveira A, Straioto FG. Importância do cirurgião-dentista em Unidade de Terapia Intensiva: avaliação multidisciplinar. Rev Assoc Paul Cir Dent. 2013;67(2):107-11.

\section{Correspondência para:}

Marcos Cezar Pomini

e-mail: marcospomini@ outlook.com

Departamento de Prótese e Periodontia

Faculdade de Odontologia de Piracicaba, UNICAMP Av. Limeira, 901 Areião

13414-903 Piracicaba/SP 